\title{
Coupled Mean Flow-Amplitude Equations for Nearly Inviscid Parametrically Driven Surface Waves
}

\author{
EDGAR KNOBLOCH, CARLOS MARTEL, AND JOSÉ M. VEGA \\ Department of Physics, University of California, Berkeley, California, USA \\ E.T.S.I. Aeronáuticos, Universidad Politécnica de Madrid, Madrid, Spain
}

\begin{abstract}
Nearly inviscid parametrically excited surface gravity-capillary waves in two-dimensional periodic domains of finite depth and both small and large aspect ratio are considered. Coupled equations describing the evolution of the amplitudes of resonant left- and right-traveling waves and their interaction with a mean flow in the bulk are derived, and the conditions for their validity established. In general the mean flow consists of an inviscid part together with a viscous streaming flow driven by a tangential stress due to an oscillating viscous boundary layer near the free surface and a tangential velocity due to a bottom boundary layer. These forcing mechanisms are important even in the limit of vanishing viscosity, and provide boundary conditions for the Navier-Stokes equation satisfied by the mean flow in the bulk. The streaming flow is responsible for several instabilities leading to pattern drift.
\end{abstract}

KEYwords: Faraday waves; streaming flow; gravity-capillary waves; parametric resonance

\section{INTRODUCTION}

Parametrically driven surface gravity-capillary waves in low viscosity fluids are traditionally discussed using a velocity potential formulation. However, this formulation has a serious shortcoming in that it ignores large-scale streaming flows that may be driven by the time-averaged Reynolds stress in the oscillatory boundary layers at the container walls, or at the free surface. We describe a systematic asymptotic technique that includes such flows and show that these flows can interact nontrivially with the waves that are in turn responsible for them. We show that this interaction can lead to new types of instability of parametrically driven waves at finite amplitude, and discuss some of their consequences. We treat only systems with periodic boundary conditions, either in two dimensions or in a cylindrical container, and discuss two cases. In the first the length of the periodic domain is large relative to the wavelength of the instability, and the mean flow contains an important inviscid contribution. In the second the wavelength and container length are comparable and the dynamics of the system is more sensitive to the container shape. In particular, if the shape is perturbed away from circular, the interaction between the streaming flow 
and the wave amplitude is enhanced, and complex dynamics may result. These conclusions generalize readily to containers of other shapes suggesting a new class of experiments.

\section{THE FARADAY SYSTEM}

Surface gravity-capillary waves or Faraday waves can be excited parametrically by the vertical oscillation of a container. ${ }^{1-3}$ We consider a container in the form of a right cylinder with horizontal cross-section $\Sigma$, filled level with the brim at $z=0$. In this geometry the contact line is pinned at the lateral boundary and complications associated with contact line dynamics are reduced. We nondimensionalize the governing equations using the unperturbed depth $h$ as unit of length and the gravity-capillary time $\left[g / h+T /\left(\rho h^{3}\right)\right]^{-1 / 2}$ as unit of time (here $g$ is the gravitational acceleration, $T$ is the coefficient of surface tension, and $\rho$ is the density), and obtain

$$
\begin{gathered}
\frac{\partial \mathbf{v}}{\partial t}-\mathbf{v} \times(\nabla \times \mathbf{v})=-\nabla \Pi+C_{g} \Delta \mathbf{v}, \nabla \cdot \mathbf{v}=0, \text { if }(x, y) \in \Sigma,-1<z<f, \\
\mathbf{v}=0 \text { if } z=-1 \text { or }(x, y) \in \partial \Sigma, f=0 \text { if }(x, y) \in \partial \Sigma, \\
\mathbf{v} \cdot \mathbf{n}=\frac{\partial f}{\partial t}\left(\mathbf{e}_{z} \cdot \mathbf{n}\right),\left[\left(\nabla \mathbf{v}+\nabla \mathbf{v}^{\mathrm{T}}\right) \cdot \mathbf{n}\right] \times \mathbf{n}=0 \text { at } z=f, \\
\Pi-\frac{|\mathbf{v}|^{2}}{2}-(1-S) f+S \nabla \cdot\left[\frac{\nabla f}{\left(1+|\nabla f|^{2}\right)^{1 / 2}}\right] \\
=C_{g}\left[\left(\nabla \mathbf{v}+\nabla \mathbf{v}^{\mathrm{T}}\right) \cdot \mathbf{n}\right] \cdot \mathbf{n}-4 \mu \omega^{2} f \cos 2 \omega t \text { at } z=f,
\end{gathered}
$$

where $\mathbf{v}$ is the velocity, $f$ is the associated vertical deflection of the free surface (constrained by volume conservation), $\Pi=p+|\mathbf{v}|^{2} / 2+(1-S) z-4 \mu \omega^{2} z \cos 2 \omega t$ is the hydrostatic stagnation pressure, $\mathbf{n}$ is the outward unit normal to the free surface, $\mathbf{e}_{\mathrm{z}}$ is the upward unit vector, and $\partial \Sigma$ denotes the boundary of the cross-section $\Sigma$ (i.e., the lateral walls). The real parameters $\mu>0$ and $2 \omega$ denote the amplitude and frequency of the forcing. The quantity $C_{g} \equiv v /\left(g h^{3}+T h / \rho\right)^{1 / 2}$, where $v$ is the kinematic viscosity, is a gravity-capillary number and $S \equiv T /\left(T+\rho g h^{2}\right)$ is a gravity-capillary balance parameter; these are related to the usual capillary number $C \equiv v \sqrt{\rho / T h}$ and Bond number $B \equiv \rho g h^{2} / T$ by

$$
C_{g}=\frac{C}{(1+B)^{1 / 2}}, S=\frac{1}{1+B} \text {. }
$$

The parameter $S$ is such that $0 \leq S \leq 1$ with $S=0$ and $S=1$ corresponding to the purely gravitational limit $(T=0)$ and the purely capillary limit $(g=0)$, respectively.

In this paper we consider the (nearly inviscid, nearly resonant, weakly nonlinear) limit

$$
C_{g} \ll 1,|\omega-\Omega| \ll<1, \mu \ll 1,
$$

where $\Omega$ is an inviscid eigenfrequency of the linearized problem around the flat state. In this case the vorticity contamination of the bulk from the boundary layers at the walls and the free surface remains negligible for times that are not too long, and the flow in the bulk is correctly described by an inviscid formulation but with 
boundary conditions determined by a boundary layer analysis. In general, this flow consists of an inviscid part and a viscous part, hereafter called the streaming flow. As discussed elsewhere ${ }^{4,5}$ the streaming flow enters into the problem because the linearized problem admits hydrodynamic (or viscous) modes, ${ }^{6}$ in addition to the usual surface modes. In the nearly inviscid limit the former decay more slowly than the surface modes, and so are easily excited, forming the streaming flow. For small $C_{g}$, these modes take the form $(\mathbf{v}, \Pi, f)=\left(\mathbf{U}, C_{g} P, C_{g} F\right) \exp \left(C_{g} \lambda t\right)+\ldots$, with the (real) eigenvalue $\lambda<0$ given by

$$
\begin{gathered}
\nabla \cdot \mathbf{U}=0, \lambda \mathbf{U}=-\nabla P+\Delta \mathbf{U} \text { if }(x, y) \in \Sigma,-1<z<0, \\
\mathbf{U}=0 \text { if } z=-1 \text { or }(x, y) \in \partial \Sigma, \\
\mathbf{e}_{z} \cdot \mathbf{U}=0,\left[\mathbf{e}_{z} \cdot\left(\nabla \mathbf{U}+\nabla \mathbf{U}^{\mathrm{T}}\right)\right] \times \mathbf{e}_{z}=0 \text { at } z=0 .
\end{gathered}
$$

The associated (scaled) free surface deflection $F$ is calculated a posteriori from the normal stress balance across $z=0$,

$$
S \Delta F-(1-S) F=\left(-P+\left[\left(\nabla \mathbf{U}+\nabla \mathbf{U}^{\mathrm{T}}\right) \cdot \mathbf{e}_{z}\right] \cdot \mathbf{e}_{z}\right)_{z=0},
$$

subject to $F=0$ on $\partial \Sigma$, and $\int_{\Sigma} F d x d y=0$. Thus, in contrast to the surface modes, the hydrodynamic modes are nonoscillatory and exhibit $O\left(C_{g}\right)$ free surface deflection. Moreover, these modes decay on an $O\left(C_{g}^{-1}\right)$ time scale, in contrast to the $O\left(C_{g}^{-1 / 2}\right)$ time scale of the surface modes, and hence cannot be ignored a priori in a weakly nonlinear theory.

\section{MODERATELY LARGE DOMAINS: $L>>1$}

In this regime it is possible to perform a multiscale analysis of the governing equations using $C_{g}, L^{-1}$, and $\mu$ as unrelated small parameters. The problem is simplest in two dimensions, where we can use a stream function formulation-that is, we write $\mathbf{v}=\left(-\psi_{\mathrm{Z}}, 0, \psi_{x}\right)$. We focus on two well-separated scales in both space $(x \sim 1$ and $x>>1)$ and time $(t \sim 1$ and $t>>1)$, and derive equations for small, slowly varying amplitudes $A$ and $B$ of left- and right-propagating waves defined by

$$
\begin{gathered}
f=e^{i \omega t}\left(A e^{i k x}+B e^{-i k x}\right)+\gamma_{1} A \bar{B} e^{2 i k x}+\gamma_{2} e^{2 i \omega t}\left(A^{2} e^{2 i k x}+B^{2} e^{-2 i k x}\right) \\
+f^{+} e^{i \omega t+i k x}+f^{-} e^{i \omega t-i k x}+\text { c.c. }+f^{m}+N R T,
\end{gathered}
$$

with similar expressions for the remaining fields. The quantities $f^{ \pm}$and $f^{m}$ represent resonant second order terms, whereas $N R T$ denotes nonresonant terms. The superscript $m$ denotes terms associated with the mean flow; $f^{m}$ depends weakly on time but may depend strongly on $x$. A systematic expansion procedure ${ }^{5}$ then leads to the equations

$$
\begin{gathered}
A_{t}-v_{g} A_{x}=i \alpha A_{x x}-(\delta+i d) A+i\left(\alpha_{3}|A|^{2}-\alpha_{4}|B|^{2}\right) A+i \alpha_{5} \mu \bar{B} \\
+i \alpha_{6} \int_{-1}^{0} g(z)\left\langle\psi_{z}^{m}\right\rangle^{x} d z A+i \alpha_{7}\left\langle f^{m}\right\rangle^{x} A, \\
B_{t}+v_{g} B_{x}=i \alpha B_{x x}-(\delta+i d) B+i\left(\alpha_{3}|B|^{2}-\alpha_{4}|A|^{2}\right) B+i \alpha_{5} \mu \bar{A} \\
-i \alpha_{6} \int_{-1}^{0} g(z)\left\langle\psi_{z}^{m}\right\rangle^{x} d z B+i \alpha_{7}\left\langle f^{m}\right\rangle^{x} B,
\end{gathered}
$$




$$
A(x+L, t) \equiv A(x, t), B(x+L, t) \equiv B(x, t) .
$$

The first seven terms in these equations, accounting for inertia, propagation at the group velocity $v_{g}$, dispersion, damping, detuning, cubic nonlinearity and parametric forcing, are familiar from weakly nonlinear, nearly inviscid theories. ${ }^{7}$ These theories lead to the expressions

$$
\begin{aligned}
& v_{g}=\omega^{\prime}(k), \alpha=-\omega^{\prime \prime}(k) / 2, \delta=\alpha_{1} C_{g}^{1 / 2}+\alpha_{2} C_{g}, \\
& \alpha_{1}=\frac{k(\omega / 2)^{1 / 2}}{\sinh 2 k}, \alpha_{2}=k^{2}\left[2+\frac{1+\sigma^{2}}{4 \sinh ^{2} k}\right] \text {, } \\
& \alpha_{3}=\frac{\omega k^{2}\left[(1-S)\left(9-\sigma^{2}\right)\left(1-\sigma^{2}\right)+S k^{2}\left(7-\sigma^{2}\right)\left(3-\sigma^{2}\right)\right]}{4 \sigma^{2}\left[(1-S) \sigma^{2}-S k^{2}\left(3-\sigma^{2}\right)\right]} \\
& +\frac{\omega k^{2}\left[8(1-S)+5 S k^{2}\right]}{4\left(1-S+S k^{2}\right)} \\
& \alpha_{4}=\frac{\omega k^{2}}{2}\left[\frac{\left(1-S+S k^{2}\right)\left(1+\sigma^{2}\right)^{2}}{\left(1-S+4 S k^{2}\right) \sigma^{2}}+\frac{4(1-S)+7 S k^{2}}{1-S+S k^{2}}\right] \text {, } \\
& \alpha_{5}=\omega k \sigma \text {, }
\end{aligned}
$$

where $\omega(k)=\left[\left(1-S+S k^{2}\right) k \sigma\right]^{1 / 2}$ is the dispersion relation and $\sigma \equiv \tanh k$, that are recovered in the present formulation. In particular, the cubic coefficients coincide with those obtained in strictly inviscid formulations. ${ }^{8-10}$ The coefficient $\alpha_{3}$ diverges at (excluded) resonant wave numbers that satisfy $\omega(2 k)=2 \omega(k)$. The detuning $d$ is given by

$$
d=\alpha_{1} C_{g}^{1 / 2}-\left(2 \pi N L^{-1}-k\right) v_{g}, N=\text { integer, }
$$

where the last term represents the mismatch between the wavelength $2 \pi / k$ selected by the forcing frequency and the domain length $L$. The last two terms in Equations (7) and (8) describe the coupling to the mean flow in the bulk (be it viscous or inviscid in origin) in terms of (a local average $\langle\cdot\rangle^{x}$ of) the stream function $\psi^{m}$ for this flow and the associated free surface elevation $f^{m}$. The coefficients of these terms and the function $g$ are given by

$$
\begin{gathered}
\alpha_{6}=k \sigma / 2 \omega, \alpha_{7}=\omega k\left(1-\sigma^{2}\right) / 2 \sigma, \\
g(z)=2 \omega k \cosh [2 k(z+1)] / \sinh ^{2} k,
\end{gathered}
$$

and are real. The new terms are, therefore, conservative, implying that at leading order the mean flow does not extract energy from the system. This result is consistent with the small steepness of the associated surface displacement and its small speed compared with the speed $|\nabla \psi|$ due to the surface waves. The mean flow variables in the bulk depend weakly on time but strongly on both $x$ and $z$, and evolve according to the equations

$$
\begin{gathered}
\Omega_{t}^{m}-\left[\psi_{z}^{m}+\left(|A|^{2}-|B|^{2}\right) g(z)\right] \Omega_{x}^{m}+\psi_{x}^{m} \Omega_{z}^{m}=C_{g}\left(\Omega_{x x}^{m}+\Omega_{z z}^{m}\right), \\
\Omega^{m}=\psi_{x x}^{m}+\psi_{z z}^{m},
\end{gathered}
$$

with 


$$
\begin{gathered}
\psi_{x}^{m}-f_{t}^{m}=\beta_{1}\left(|B|^{2}-|A|^{2}\right)_{x}, \psi_{z z}^{m}=\beta_{2}\left(|A|^{2}-|B|^{2}\right), \\
(1-S) f_{x}^{m}-S f_{x x x}^{m}-\psi_{z t}^{m}+C_{g}\left(\psi_{z z z}^{m}+3 \psi_{x x z}^{m}\right)=-\beta_{3}\left(|A|^{2}+|B|^{2}\right)_{x} \text { at } z=0
\end{gathered}
$$

and

$$
\begin{gathered}
\psi_{z}^{m}=-\beta_{4}\left[i A \bar{B} e^{2 i k x}+\text { c.c. }+|B|^{2}-|A|^{2}\right], \\
\int_{0}^{L} \Omega_{z}^{m} d x=\psi^{m}=0 \text { at } z=-1
\end{gathered}
$$

Also, $\psi^{m}(x+L, z, t) \equiv \psi^{m}(x, z, t), f^{m}(x+L, t) \equiv f^{m}(x, t)$, subject to $\int_{0}^{L} f^{m}(x, t) d x=0$. Here, $\beta_{1}=2 \omega / \sigma, \beta_{2}=8 \omega k^{2} / \sigma, \beta_{3}=\left(1-\sigma^{2}\right) \omega^{2} / \sigma^{2}, \beta_{4}=3\left(1-\sigma^{2}\right) \omega k / \sigma^{2}$. Thus, the mean flow is forced by the surface waves in two ways. The right sides of the boundary conditions (12a) and $\mathbf{( 1 2 c )}$ provide a normal forcing mechanism; this mechanism is the only one present in strictly inviscid theory 9,11 and does not appear unless the aspect ratio is large. The right sides of the boundary conditions (12b) and (13a) describe two shear forcing mechanisms, a tangential stress at the free surface ${ }^{12}$ and a tangential velocity at the bottom wall. ${ }^{13}$ Note that neither of these forcing terms vanishes in the limit of small viscosity (i.e., as $C_{g} \rightarrow 0$ ). The shear nature of these forcing terms leads us to retain the viscous term in (11a) even when $C_{g}$ is quite small. In fact, when $C_{g}$ is very small, the effective Reynolds number of the mean flow is quite large. Thus, the mean flow itself generates additional boundary layers near the top and bottom of the container, and these must be thicker than the original boundary layers for the validity of the analysis. This puts an additional restriction on the validity of the equations. ${ }^{5}$ There is a third, less effective but inviscid, volumetric forcing mechanism associated with the second term in the vorticity equation (11a) that looks like a horizontal force $\left(|A|^{2}-|B|^{2}\right) g(z) \Omega^{m}$ and is sometimes called the vortex force. Although this term vanishes in the absence of mean flow, it can change the stability properties of the flow and enhance or limit the effect of the remaining forcing terms.

In the following we refer to Equations (7)-(9) and (11)-(13) as the general coupled amplitude-mean-flow (GCAMF) equations. These equations differ from the exact equations forming the starting point for the analysis in three essential simplifications: the fast oscillations associated with the surface waves have been filtered out, the effect of the thin primary viscous boundary layers is replaced by effective boundary conditions on the flow in the bulk-viz. (12b) and (13a) - and the surface boundary conditions are applied at the unperturbed location of the free surface-viz. $z=0$. Thus, only the much broader (secondary) boundary layers associated with the (slowly varying) streaming flow need to be resolved in any numerical simulation.

\section{GRAVITY-CAPILLARY WAVES IN MODERATELY LARGE ASPECT RATIO CONTAINERS}

The GCAMF equations describe small amplitude slowly varying wavetrains whenever the parameters $C_{g}, L^{-1}$, and $\boldsymbol{\mu}$ are small, but otherwise unrelated to one another. Any relation between them will therefore lead to further simplification. To derive such simplified equations we consider the distinguished limit (see the shaded region in FIGURE 1A). 

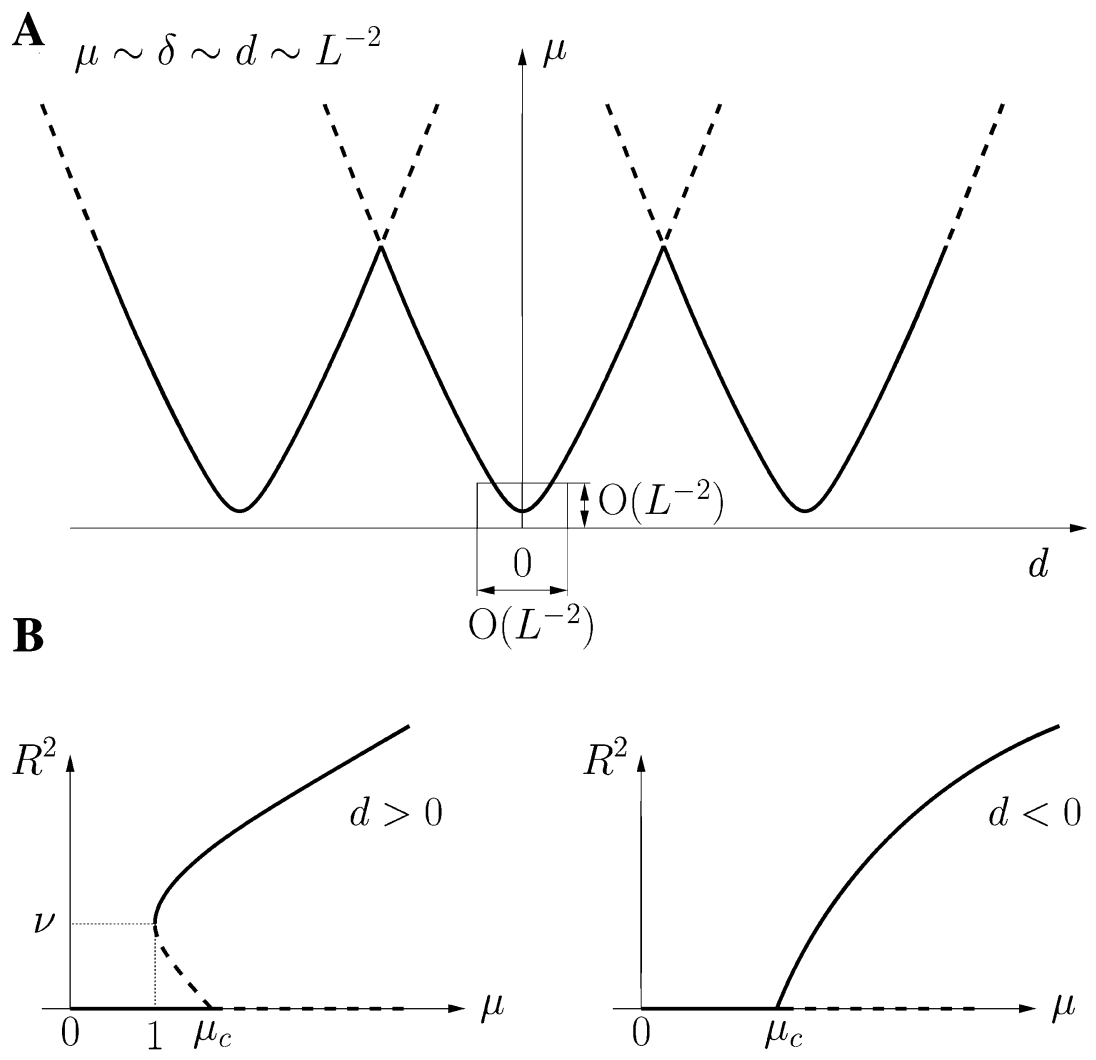

FIGURE 1. (A) Sketch of the primary resonance tongue in the scaling regime of interest. (B) Bifurcation diagrams for the resulting standing waves near onset.

$$
\frac{\delta L^{2}}{\alpha}=\Delta \sim 1, \frac{d L^{2}}{\alpha}=D \sim 1, \frac{\mu L^{2}}{\alpha} \equiv M \sim 1,
$$

with $k=O(1)$ and $\left|\ln C_{g}\right|=O(1)$. The simplified equations will then be formally valid for $1<<L<C_{g}^{-1 / 2}$ if $k \sim 1$. These are derived under the assumption $1-S \sim 1$ using a multiple scale method with $x$ and $t$ as fast variables and

$$
\zeta \equiv \frac{x}{L}, \tau \equiv \frac{t}{L}, T \equiv \frac{t}{L^{2}}
$$

as slow variables. In terms of these variables the local horizontal average $\langle\cdot\rangle^{x}$ becomes an average over the fast variable $x$. Note that assumption (14) imposes an implicit relation between $L$ and $C_{g}$. When $1-S \sim 1$ the nearly inviscid and viscous mean flows can be clearly distinguished from one another and the viscous mean flow can be identified by taking appropriate averages of the entire mean flow over the intermediate time scale $\tau$, that is, the mean flow variables $\psi^{m}, \Omega^{m}$, and $f^{m}$ take the form 


$$
\begin{aligned}
& \psi^{m}(x, z, \zeta, \tau, T)=\psi^{v}(x, z, \zeta, T)+\psi^{i}(x, z, \zeta, \tau, T), \\
& \Omega^{m}(x, z, \zeta, \tau, T)=\Omega^{v}(x, z, \zeta, T)+\Omega^{i}(x, z, \zeta, \tau, T), \\
& f^{m}(x, \zeta, \tau, T)=f^{v}(x, \zeta, T)+f^{i}(x, \zeta, \tau, T),
\end{aligned}
$$

with integrals over $\tau$ of $\psi_{x}^{i}, \psi_{\zeta}^{i}, \psi_{z}^{i}, \Omega^{i}$, and $f^{i}$ required to be bounded as $\tau \rightarrow \infty$. Thus, the nearly inviscid mean flow is purely oscillatory on the time scale $\tau$. Since its frequency is of the order of $L^{-1}$ (see Eq. (15)), which is large compared with $C_{g}$, the inertial term for this flow is large in comparison with the viscous terms (see Eq. (11)), except in two secondary boundary layers, of thickness of the order of $\left(C_{g} L\right)^{1 / 2}(<<1)$, attached to the bottom plate and the free surface. Note that, as required for the consistency of the analysis, these boundary layers are much thicker than the primary boundary layers associated with the surface waves (see FIGURE 2), which provide the boundary conditions (12) and (13) for the mean flow. Moreover, the width of these secondary boundary layers remains small as $\tau \rightarrow \infty$ and (to leading order) the vorticity of this nearly inviscid mean flow remains confined to these boundary layers. Note that without the requirement that the inviscid flow be purely oscillatory, the vorticity would diffuse out of these boundary layers and affect the structure of the whole nearly inviscid solution even at leading order. In fact, vorticity does diffuse (and is convected) away from the boundary layers, but this vorticity transport is included in the viscous mean flow. The vorticity associated with the nearly inviscid mean flow is at most of the order of

$$
\left.|| A\right|^{2}-|B|^{2} \mid \text { and }\left(|A|^{2}+|B|^{2}\right)\left(C_{g} L\right)^{-1 / 2}
$$

in the upper and lower secondary boundary layers, respectively; the jump in the associated stream function $\psi^{i}$ across each boundary layer is $O\left(C_{g} L\right)$ times smaller, and affects only higher order terms; as a consequence the secondary boundary layers

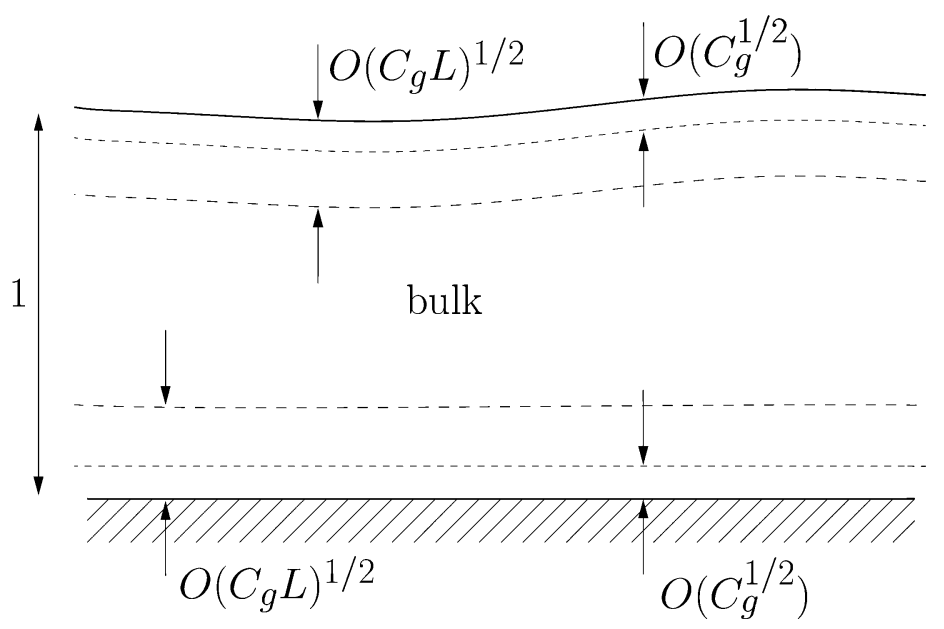

FIGURE 2. Sketch of the primary and secondary boundary layers, indicating their widths in comparison with the layer depth. 
can be completely ignored and no additional contributions to the boundary conditions on the nearly inviscid flow need be included in (12) and (13). Outside of these boundary layers, the complex amplitudes and the flow variables associated with the nearly inviscid mean flow are expanded as

$$
\begin{aligned}
& (A, B)=L^{-1}\left(A_{0}, B_{0}\right)+L^{-2}\left(A_{1}, B_{1}\right)+\ldots, \\
& \left(\psi^{v}, \Omega^{v}\right)=L^{-2}\left(\phi_{0}^{v}, W_{0}^{v}\right)+\ldots, f^{v}=L^{-3} F_{0}^{v}+\ldots, \\
& \left(\psi^{i}, f^{i}\right)=L^{-2}\left(\phi_{0}^{i}, F_{0}^{i}\right)+L^{-3}\left(\phi_{1}^{i}, F_{1}^{i}\right)+\ldots, \\
& \Omega^{i}=L^{-3} W_{0}^{i}+\ldots .
\end{aligned}
$$

Substitution of (14)-(16) and (18) into (7)-(13) leads to the following:

(1) From (11)-(13), at leading order,

$$
\begin{gathered}
\phi_{0 x x}^{i}+\phi_{0 z z}^{i}=0 \text { in }-1<z<0, \\
\phi_{0}^{i}=0 \text { at } z=-1, \phi_{0 x}^{i}=0 \text { at } z=0,
\end{gathered}
$$

together with $F_{0 x}^{i}=0$. Thus,

$$
\phi_{0}^{i}=(z+1) \Phi_{0}^{i}(\zeta, \tau, T), F_{0}^{i}=F_{0}^{i}(\zeta, \tau, T) .
$$

At second order, the boundary conditions $(\mathbf{1 2 a})$ and (12c) yield

$$
\begin{aligned}
\phi_{1 x}^{i}(x, 0, \zeta, \tau, T) & =F_{0 \tau}^{i}-\Phi_{0 \zeta}^{i}+\beta_{1}\left(\left|B_{0}\right|^{2}-\left|A_{0}\right|^{2}\right)_{\zeta} \\
(1-S) F_{1 x}^{i}-S F_{1 x x x}^{i} & =\Phi_{0 \tau}^{i}-(1-S) F_{0 \zeta}^{i}-\beta_{3}\left(\left|A_{0}\right|^{2}+\left|B_{0}\right|^{2}\right)_{\zeta}
\end{aligned}
$$

at $z=0$. Since the right hand sides of these two equations are independent of the fast variable $x$ and both $\phi_{1}^{i}$ and $F_{1}^{i}$ must be bounded in $x$, it follows that

$$
\begin{gathered}
\Phi_{0 \zeta}^{i}-F_{0 \tau}^{i}=\beta_{1}\left(\left|B_{0}\right|^{2}-\left|A_{0}\right|^{2}\right)_{\zeta}, \\
\Phi_{0 \tau}^{i}-v_{p}^{2} F_{0 \zeta}^{i}=\beta_{3}\left(\left|A_{0}\right|^{2}+\left|B_{0}\right|^{2}\right)_{\zeta}
\end{gathered}
$$

where

$$
v_{p}=(1-S)^{1 / 2}
$$

is the phase velocity of long wavelength surface gravity waves. Equations (20) must be integrated with the following additional conditions

$$
\Phi_{0}^{i}(\zeta+1, \tau, T) \equiv \Phi_{0}^{i}(\zeta, \tau, T), F_{0}^{i}(\zeta+1, \tau, T) \equiv F_{0}^{i}(\zeta, \tau, T),
$$

and the requirements that integrals over $\tau$ of $\Phi_{0 \zeta}^{i}$ and $F_{0}^{i}$ remain bounded as $\tau \rightarrow \infty$, with $\int_{0}^{1} F_{0}^{i} d \zeta=0$.

(2) The leading order contributions to Equations (7) and (8) yield

$$
A_{0 \tau}-v_{g} A_{0 \zeta}=B_{0 \tau}+v_{g} B_{0 \zeta}=0 \text {. }
$$

Thus,

$$
A_{0}=A_{0}(\xi, T), B_{0}=B_{0}(\eta, T),
$$

where $\xi$ and $\eta$ are the characteristic variables

$$
\xi=\zeta+v_{g} \tau, \eta=\zeta-v_{g} \tau
$$

Moreover, according to (9), 


$$
A_{0}(\xi+1, T) \equiv A_{0}(\xi, T), B_{0}(\eta+1, T) \equiv B_{0}(\eta, T) .
$$

Substitution of these expressions into $(\mathbf{2 0})$ followed by integration of the resulting equations yields

$$
\begin{gathered}
\Phi_{0}^{i}=\frac{\beta_{1} v_{p}^{2}+\beta_{3} v_{g}}{v_{g}^{2}-v_{p}^{2}}\left[\left|A_{0}\right|^{2}-\left|B_{0}\right|^{2}-\left\langle\left|A_{0}\right|^{2}-\left|B_{0}\right|^{2}\right\rangle \zeta\right] \\
\quad+v_{p}\left[F^{+}\left(\zeta+v_{p} \tau, T\right)-F^{-}\left(\zeta-v_{p} \tau, T\right)\right], \\
F_{0}^{i}=\frac{\beta_{1} v_{g}+\beta_{3}}{v_{g}^{2}-v_{p}^{2}}\left[\left|A_{0}\right|^{2}+\left|B_{0}\right|^{2}-\left\langle\left|A_{0}\right|^{2}+\left|B_{0}\right|^{2}\right\rangle\right] \\
+F^{+}\left(\zeta+v_{p} \tau, T\right)+F^{-}\left(\zeta-v_{p} \tau, T\right),
\end{gathered}
$$

where $\langle\cdot\rangle^{\zeta}$ denotes the mean value in the slow spatial variable $\zeta$, that is,

$$
\langle G\rangle \zeta=\int_{0}^{1} G d \zeta
$$

and the functions $F^{ \pm}$are such that

$$
F^{ \pm}\left(\zeta+1 \pm v_{p} \tau, T\right) \equiv F^{ \pm}\left(\zeta \pm v_{p} \tau, T\right),\left\langle F^{ \pm}\right\rangle \zeta=0
$$

The particular solution of (26) and (27) yields the usual inviscid mean flow included in nearly inviscid theories; ${ }^{9}$ the averaged terms are a consequence of volume conservation $^{9}$ and the requirement that the nearly inviscid mean flow has a zero mean on the time scale $\tau$; the latter condition is never imposed in strictly inviscid theories but is essential in the limit we are considering, as explained above. To avoid the breakdown of the solution (26) and (27) at $v_{p}=v_{g}$ we assume that

$$
\left|v_{p}-v_{g}\right| \sim 1 \text {. }
$$

The functions $F^{ \pm}$remain undetermined at this stage. In fact, they are not needed below because the evolution of both the viscous mean flow and of the complex amplitudes is decoupled from these functions. However, at next order one finds that $F^{ \pm}$remain constant on the time scale $T$, but decay exponentially due to viscous effects (resulting from viscous dissipation in the secondary boundary layer attached to the bottom plate) on the time scale $t \sim\left(L / C_{g}\right)^{1 / 2}$.

(3) The evolution equations for $A_{0}$ and $B_{0}$ on the time scale $T$ are readily obtained from Equations (7)-(9), invoking (14), (16), (26), (27), (29), and eliminating secular terms (i.e., requiring $\left|A_{1}\right|$ and $\left|B_{1}\right|$ to be bounded on the time scale $\tau$ ):

$$
\begin{gathered}
A_{0 T}=i \alpha A_{0 \xi \xi}-(\Delta+i D) A_{0}+i\left[\left(\alpha_{3}+\alpha_{8}\right)\left|A_{0}\right|^{2}-\alpha_{8}\left\langle\left|A_{0}\right|^{2}\right\rangle^{\xi}-\alpha_{4}\left\langle\left|B_{0}\right|^{2}\right\rangle^{\eta}\right] A_{0} \\
+i \alpha_{5} M\left\langle\overline{B_{0}}\right\rangle^{\eta}+\left.i \alpha_{6}\right|_{-1} ^{0} g(z)\left\langle\left\langle\phi_{0 z}^{v}\right\rangle^{x}\right\rangle^{\zeta} d z A_{0} \\
B_{0 T}=i \alpha B_{0 \eta \eta}-(\Delta+i D) \\
B_{0}+i\left[\left(\alpha_{3}+\alpha_{8}\right)\left|B_{0}\right|^{2}-\alpha_{8}\left\langle\left|B_{0}\right|^{2}\right\rangle^{\eta}-\alpha_{4}\left\langle\left|A_{0}\right|^{2}\right\rangle^{\xi}\right] B_{0} \\
+i \alpha_{5} M\left\langle\overline{A_{0}}\right\rangle^{\xi}-i \alpha_{6} \int_{-1}^{0} g(z)\left\langle\left\langle\phi_{0 z}^{v}\right\rangle^{x}\right\rangle^{\zeta} d z B_{0}
\end{gathered}
$$

subject to (25). Here $\xi$ and $\eta$ are the comoving variables defined in (24), and $\langle\cdot\rangle^{x}$, $\langle\cdot\rangle^{\zeta},\langle\cdot\rangle^{\xi}$, and $\langle\cdot\rangle^{\eta}$ denote mean values over the variables $x, \zeta, \xi$, and $\eta$, respectively. Note that $\zeta$ averages over functions of $A_{0}$ are equivalent to $\xi$ averages, whereas those over functions of $B_{0}$ are equivalent to $\eta$ averages.

The real coefficient $\alpha_{8}$ is given by 


$$
\alpha_{8}=\frac{\alpha_{6}(2 \omega / \sigma)\left(\beta_{1} v_{p}^{2}+\beta_{3} v_{g}\right)+\alpha_{7}\left(\beta_{1} v_{g}+\beta_{3}\right)}{v_{g}^{2}-v_{p}^{2}} .
$$

Equations (31) are independent of $F^{ \pm}$because of the second condition in (29).

When $\Delta>0$ Equations (31) can be used to show that

$$
\left\langle\left|A_{0}\right|^{2}-\left|B_{0}\right|^{2}\right\rangle^{\tau}=\left\langle\left|A_{0}\right|^{2}\right\rangle^{\xi}-\left\langle\left|B_{0}\right|^{2}\right\rangle^{\eta} \rightarrow 0 \text { as } T \rightarrow \infty
$$

and the result used to simplify the equations for the viscous mean flow in the long time limit:

$$
\begin{gathered}
W_{0 T}^{v}-\phi_{0 z}^{v} W_{0 x}^{v}+\phi_{0 x}^{v} W_{0 z}^{v}=R e^{-1}\left(W_{0 x x}^{v}+W_{0 z z}^{v}\right), \\
\phi_{0 x x}^{v}+\phi_{0 z z}^{v}=W_{0}^{v} \text { in }-1<z<0, \\
\phi_{0 x}^{v}=\phi_{0 z z}^{v}=0 \text { at } z=0, \\
\left\langle\left\langle W_{0 z}^{v}\right\rangle^{x}\right\rangle \zeta=\phi_{0}^{v}=0, \phi_{0 z}^{v}=-\beta_{4}\left[i\left\langle A_{0} \bar{B}_{0}\right\rangle^{\tau} e^{2 i k x}+\text { c.c. }\right] \text { at } z=-1, \\
\phi_{0}^{v}(x+L, \zeta+1, z, T) \equiv \phi_{0}^{v}(x, \zeta, z, T),
\end{gathered}
$$

where

$$
\operatorname{Re}=\left(C_{g} L^{2}\right)^{-1}
$$

is the effective Reynolds number associated with this flow.

Several remarks about these equations and boundary conditions are now in order. 1. The viscous mean flow is driven by the short gravity-capillary waves through the inhomogeneous term in the boundary condition (35c). Since $\left\langle A_{0} \bar{B}_{0}\right\rangle^{\tau}$ depends on both $\zeta$ and $T$ (unless either $A_{0}$ or $B_{0}$ is spatially uniform) the boundary condition implies that $\phi_{0}^{v}$ (and hence $W_{0}^{v}$ ) depends on both the fast and slow horizontal spatial variables $x$ and $\zeta$. This dependence cannot be obtained in closed form, and one must, therefore, resort to numerical computations for realistically large values of $L$. 2. Higher order oscillatory terms omitted from the boundary condition $(\mathbf{3 5 c})$ oscillate on the intermediate time scale $\tau$, and hence generate secondary boundary layers. However, the contributions from these boundary layers are all subdominant and have no effect on the streaming flow at leading order. Moreover, the freesurface deflection accompanying the viscous mean flow is also small, $f^{v} \sim L^{-3}$ (see Eq. (18)), and so plays no role in the evolution of this flow, as expected of a flow involving the excitation of viscous modes.

3. The dominant forcing of the viscous mean flow comes from the lower boundary. This forcing vanishes exponentially when $k>1$ leaving only a narrow range of wave numbers within which such a mean flow is forced when $\delta=O\left(C_{g}\right) .{ }^{5}$ Thus, in most cases in which a viscous mean flow is present, one may assume that $\delta=O\left(C_{g}{ }^{1 / 2}\right)$. Note, however, that in fully three-dimensional situations ${ }^{14}$ in which lateral walls are included a viscous mean flow will be present even when $k>1$ because the forcing of the mean flow in the oscillatory boundary layers along these walls remains.

4. According to the scaling (14) and the definition of $\delta$ the effective Reynolds number $R e$ is large, and ranges from logarithmically large values if $k-\left|\ln C_{g}\right|$ to $O\left(C_{g}{ }^{-1 / 2}\right)$ if $k \sim 1$. However, even in the latter limit we must retain the viscous terms 
in (33a) in order to account for the boundary conditions (34b) and (35c). Of course, if $R e \gg 1$ vorticity diffusion is likely to be confined to thin layers, but the structure and location of all these layers cannot be anticipated in any obvious way, and one must again rely on numerical computations.

5. Note that the change of variables

$$
A_{0}=\tilde{A}_{0} e^{-i k \theta}, B_{0}=\tilde{B}_{0} e^{i k \theta}
$$

where

$$
\theta^{\prime}(T)=-\alpha_{6} k^{-1} \int_{-1}^{0} g(z)\left\langle\left\langle\phi_{0 z}^{\nu}\right\rangle^{x}\right\rangle^{\zeta} d z
$$

reduces Equations (31) to the much simpler form

$$
\begin{aligned}
& \tilde{A}_{0 T}= i \alpha \tilde{A}_{0 \xi \xi}-(\Delta+i D) \tilde{A}_{0} \\
&+i\left[\left(\alpha_{3}+\alpha_{8}\right)\left|\tilde{A}_{0}\right|^{2}-\left(\alpha_{4}+\alpha_{8}\right)\left\langle\left|\tilde{B}_{0}\right|^{2}\right\rangle^{\eta}\right] \tilde{A}_{0}+i \alpha_{5} M\left\langle\tilde{\tilde{B}}_{0}\right\rangle^{\eta}, \\
& \tilde{B}_{0 T}= i \alpha \tilde{B}_{0 \eta \eta}-(\Delta+i D) \tilde{B}_{0} \\
&+i\left[\left(\alpha_{3}+\alpha_{8}\right)\left|\tilde{B}_{0}\right|^{2}-\left(\alpha_{4}+\alpha_{8}\right)\left\langle\left|\tilde{A}_{0}\right|^{2}\right\rangle^{\xi}\right] \tilde{B}_{0}+i \alpha_{5} M\left\langle\tilde{\tilde{A}}_{0}\right\rangle^{\xi}, \\
& \tilde{A}_{0}(\xi+1, T) \equiv \tilde{A}_{0}(\xi, T), \tilde{B}_{0}(\eta+1, T) \equiv \tilde{B}_{0}(\eta, T),
\end{aligned}
$$

from which the mean flow is absent. This decoupling is a special property of the regime defined by Equation (14), but is not unique to it, as discussed further below. The resulting equations provide perhaps the simplest description of the Faraday system at large aspect ratio, and it is for this reason that they have been extensively studied. ${ }^{15}$ FIGURE 3 A shows a typical bifurcation diagram obtained from (40) when $D=0$; FIGURE $3 \mathrm{~B}$ shows the corresponding results obtained for standing waves, that is, imposing the requirement that $\tilde{A}_{0}=\tilde{B}_{0}\left(=\tilde{C}_{0}\right.$, say) identically. FIGURE $1 \mathrm{~B}$ sketches the consequence of including a small but nonzero detuning. These diagrams show the $L^{2}$ norms of $\tilde{A}_{0}, \tilde{B}_{0}$, or $\tilde{C}_{0}$ (after transients have died out) at successive intersections of a trajectory with the hypersurfaces

$$
\left\|\tilde{A}_{0}\right\|_{L^{2}}^{2}+\left\|\tilde{B}_{0}\right\|_{L^{2}}^{2}=\mu\left(\left\langle\overline{\tilde{A}}_{0}\right\rangle\left\langle\overline{\tilde{B}}_{0}\right\rangle+\text { c.c. }\right) \text {, and }\left\|\tilde{C}_{0}\right\|_{L^{2}}^{2}=\frac{1}{2} \mu\left(\left\langle\overline{\tilde{C}_{0}}\right\rangle^{2}+\text { c.c. }\right),
$$

respectively. A finite number of points therefore indicates a steady or a periodic solution, whereas scattered points indicate a chaotic trajectory. The origin of such amplitude chaos is discussed elsewhere. ${ }^{16}$ In both cases the primary instability is to spatially uniform standing waves (shown by a dashed line when unstable), followed by a supercritical secondary bifurcation to a pattern of spatially nonuniform standing waves, and then a Hopf bifurcation. However, subsequent bifurcations differ as instabilities that break the equality $\tilde{A}_{0}=\tilde{B}_{0}$ set in, producing stable waves that are not standing. As discussed elsewhere ${ }^{15}$ instabilities that break the equality $\tilde{A}_{0}=\tilde{B}_{0}$ are also responsible for introducing a type of drift into the dynamics, but the origin of this drift is entirely different from that discussed below, being a drift in the temporal phase of the oscillations in contrast to a drift in the spatial phase. The latter drift is described by the decoupled phase-mean flow equations, (33)-(36) and (39) that can exhibit complex dynamics in their own right (see below); this drift can be present regardless of whether $\tilde{A}_{0}=\tilde{B}_{0}$ or not. 

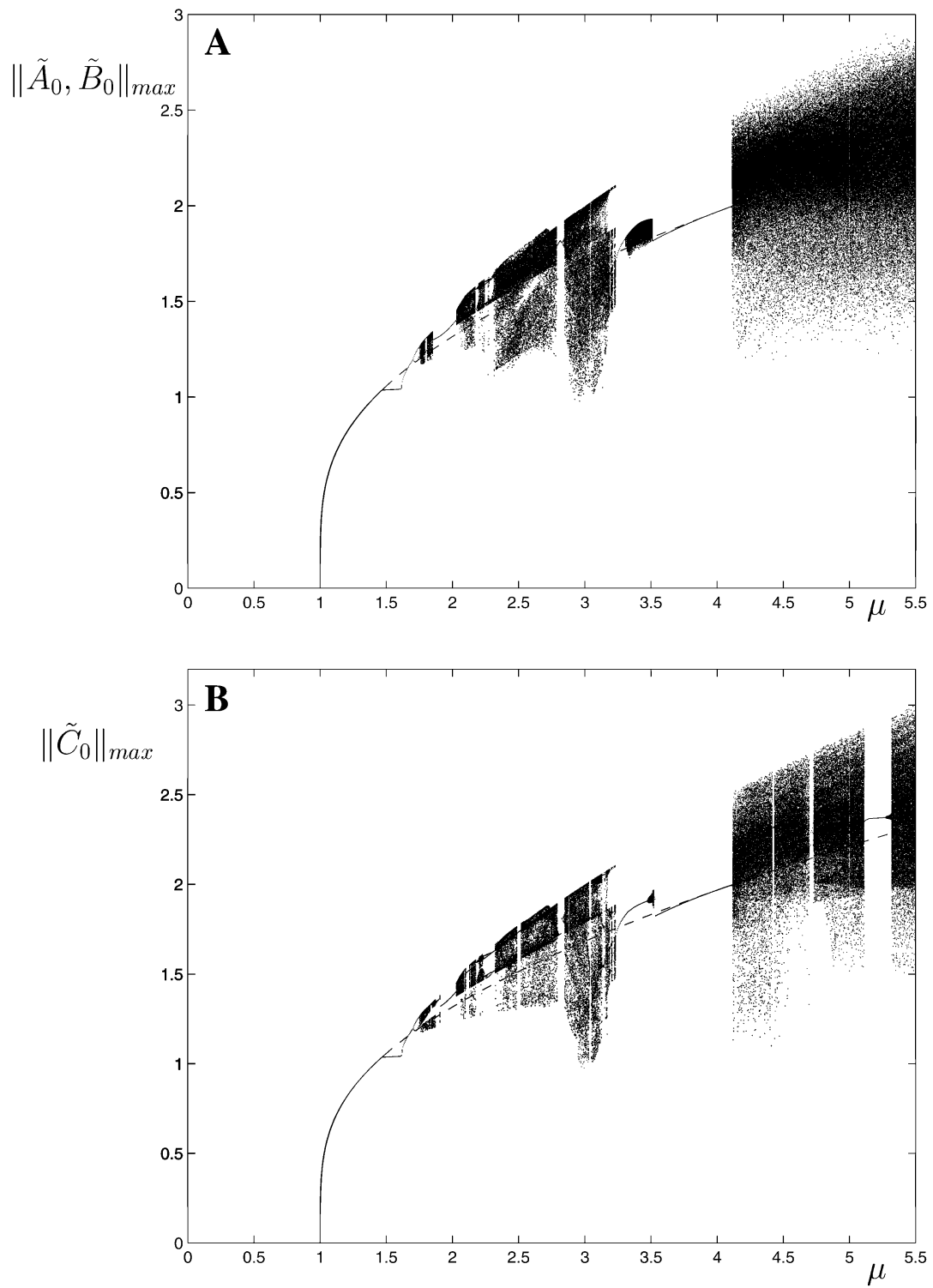

FIGURE 3. Bifurcation diagrams for Equations (40) showing successive maxima of the $L^{2}$ norm as a function of $\mu \equiv \alpha_{5} M$ when $\Delta=1, D=0, \alpha=0.1, \alpha_{3}+\alpha_{8}=1.5$, and $\alpha_{4}+\alpha_{8}=0.5$. After Martel et al. ${ }^{15}$ 


\section{SMALL DOMAINS: $L \sim 1$}

The theory described above simplifies substantially in small domains, because of the absence of the slow spatial scale $\zeta$ and the advection time scale $\tau$. Although the basic set up of the calculation is now quite different, the results are (almost) identical to those just described. This time ${ }^{17}$

$$
\begin{aligned}
& A_{t}=-(\delta+i d) A+i\left(\alpha_{3}|A|^{2}-\alpha_{4}|B|^{2}\right) A+i \alpha_{5} \mu \bar{B}-i \alpha_{6} L^{-1} \int_{-1}^{0} \int_{0}^{L} g(z) u^{v} d x d z A, \\
& B_{t}=-(\delta+i d) B+i\left(\alpha_{3}|B|^{2}-\alpha_{4}|A|^{2}\right) B+i \alpha_{5} \mu \bar{A}+i \alpha_{6} L^{-1} \int_{-1}^{0} \int_{0}^{L} g(z) u^{v} d x d z B,
\end{aligned}
$$

with $A$ and $B$ spatially constant and the coefficients given by expressions that are identical to those in (7) and (8). In these equations $t$ denotes a slow time whose magnitude is determined by the damping $\delta>0$ and the detuning $d$, both assumed to be of the same order as the forcing amplitude $\mu$; in the long time limit $|A|^{2}=|B|^{2}=R^{2}$. It follows that the mean flow $\left(u^{v}(x, z, t), w^{v}(x, z, t)\right)$ is now entirely viscous in origin, and obeys a two-dimensional Navier-Stokes equation of the form (33). If we absorb the standing wave amplitude $R$ (and some other constants) in the definition of the Reynolds number

$$
R e \equiv 2 \beta_{4} R^{2} / C_{g},
$$

this equation is to be solved subject to the boundary conditions

$$
\begin{gathered}
u^{v}=-\sin [2 k(x-\theta)], w^{v}=0 \text { at } z=-1, \\
u_{z}^{v}=0, w^{v}=0 \text { at } z=0 .
\end{gathered}
$$

Using the dimensional values of the amplitude of the waves $A_{d}$, frequency $\omega_{d}$, wave number $k_{d}$, kinematic viscosity $v$, and container depth $h$, the Reynolds number (42) of the streaming flow can also be written as

$$
R e=\frac{\omega_{d} A_{d}^{2}}{v} \frac{6 k_{d} h}{\sinh ^{2}\left(k_{d} h\right)} .
$$

Because the structure of Equations (41) is identical to that of Equations (31) the change of variables (38) leads to a decoupling of the amplitudes from the spatial phase $\theta$, which now satisfies the equation

$$
\theta_{t}=\frac{\alpha_{6}}{k L} \int_{-1}^{0} \int_{0}^{L} g(z) u^{v}(x, z, t) d x d z
$$

FIGURE 4 summarizes the solutions of this coupled phase-streaming flow problem as a function of the wave amplitude $R$, showing the maxima and minima in the drift speed $\theta_{t}$ as a function of the Reynolds number (42) for $k=2.37$ and $L \equiv 2 \pi / k=2.65$. The primary solution that sets in at $\boldsymbol{\mu}=\boldsymbol{\mu}_{c}$ consists of stable standing waves with $|A|^{2}=|B|^{2}=R^{2}$, defined up to a constant spatial phase $\theta$. The streamlines of the associated streaming flow are shown in FIGURE $5 \mathrm{~A}$ for $R e=260$, and take the form of an array of reflection-symmetric counter-rotating eddies with spatial period $L / 2$. Such reflection-symmetric states do not drift: $\theta_{t} \equiv 0$. At $R e=R e^{1} \approx 270$ this reflectionsymmetric state loses stability at a (secondary) symmetry-breaking Hopf bifurcation that produces direction-reversing waves. ${ }^{18}$ In this state the standing waves drift periodically, first to the left and then to the right, with no net displacement, but the 


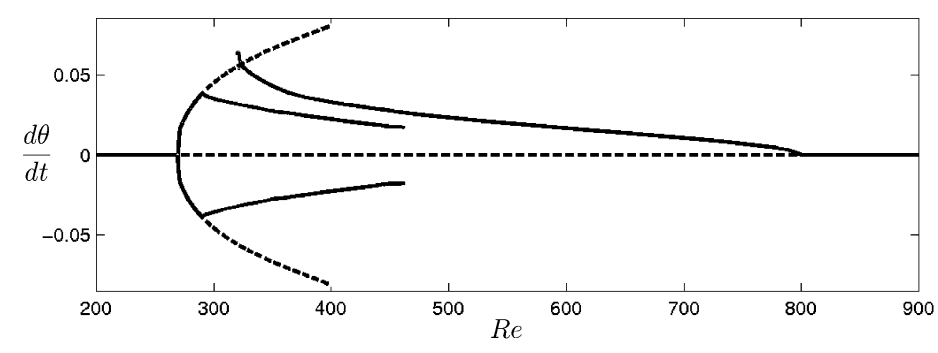

FIGURE 4. Bifurcation diagram for the coupled phase-streaming flow equations showing the drift speed $d \theta / d t$ as a function of the Reynolds number (42) for $k=2.37$ $(L=2.65)$. The secondary Hopf and parity-breaking bifurcations occur at $R e \approx 270$ and $R e \approx 800$, respectively.
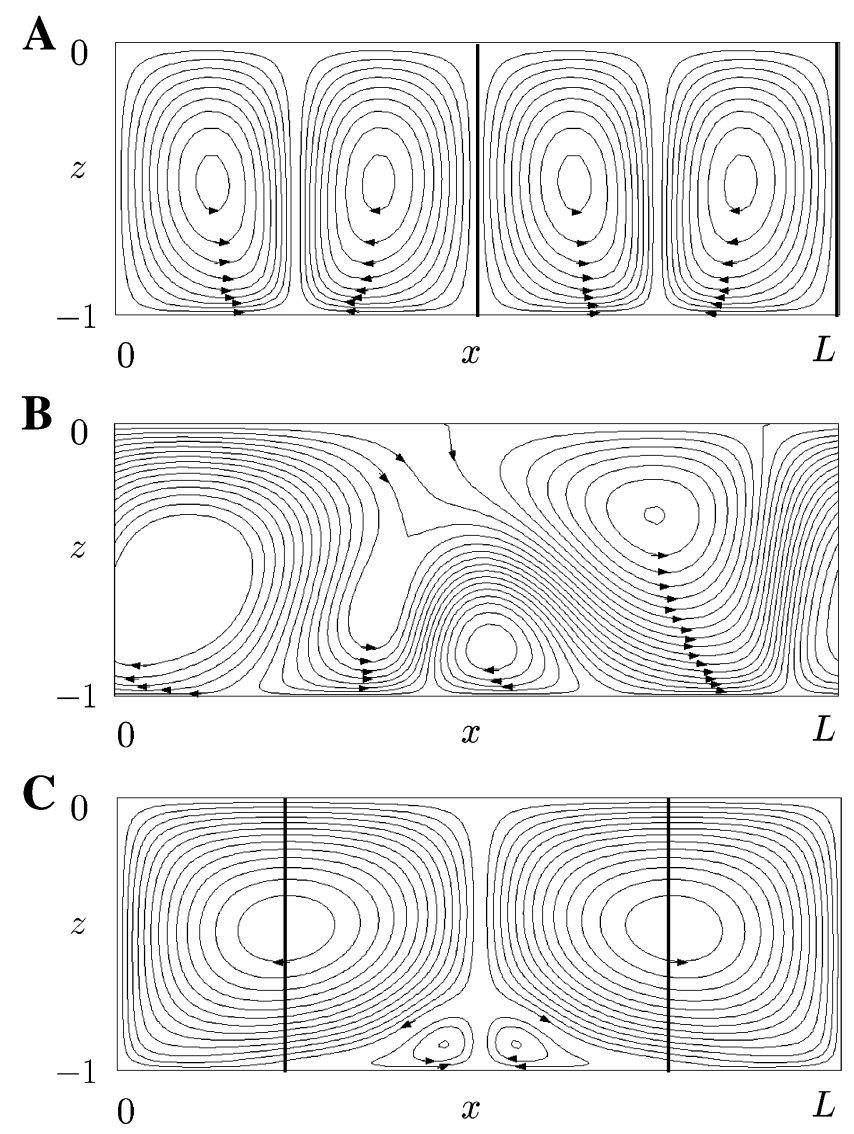

FIGURE 5. Streamlines of the streaming flow when (A) $R e=260,(\mathbf{B}) \operatorname{Re}=325$, and (C) $R e=850$. Vertical lines indicate the location of the nodes of the standing wave. 
spatial period $L / 2$ of the standing waves is preserved. This bifurcation is supercritical and the direction-reversing waves are, therefore, stable.

The onset of this instability is insensitive to the domain length $L$ once this is large enough. These oscillations lose stability at $R e \approx 291.5$ to a new family of stable direction-reversing waves, this time with spatial period $L$. These new solutions in turn become unstable at $R e \approx 466$ where a subcritical bifurcation takes the system to a new branch of steadily drifting solutions. These solutions drift either to the left or to the right, and are stable in the interval $323 \approx R e^{3}<R e<R e^{2} \approx 800$. In FIGURE 4 only the waves drifting to the right $\left(\theta_{t}>0\right)$ are shown; the corresponding streaming flow streamlines are shown (in the comoving frame) in FIGURE 5B. The bifurcation at $R e=R e^{2}$ is a parity-breaking steady state bifurcation; at this bifurcation the drift ceases, and a reflection-symmetric streaming flow is restored, but now with spatial period $L$ (FIG. 5C). This flow is stable, but in FIGURE 4 is projected on top of the unstable original symmetric state with period $L / 2$. For larger $L$ (e.g., $L=4 \pi / k$ ) several different stable states coexist, some of which lack reflection symmetry, and exhibit complex time-dependence. ${ }^{17}$ Note that neither type of drift is associated with the loss of reflection-symmetry of the waves themselves, at least at leading order; instead they are due to a symmetry-breaking instability of the associated streaming flow.

\section{EXCITATION OF STREAMING FLOWS IN NEARLY CIRCULAR DOMAINS}

The results just described are characteristic of domains with periodic boundary conditions, such as circular domains. In such domains the amplitudes decouple from the spatial phase and the streaming flow only causes drift motion in an otherwise standing wave pattern. However, the instabilities found set in only at sufficiently large Reynolds number, necessitating a numerical solution of the coupled phasestreaming flow problem. There is a simple way, however, of pushing these instabilities to much smaller amplitudes and, hence, into a regime where analytic progress is possible. This occurs as soon as the translation (rotation) invariance of the container is broken (eg., by deforming it slightly), thereby forcing the wave amplitudes to couple to the spatial phase. In systems undergoing a symmetry-breaking primary Hopf bifurcation (but with no parametric forcing) this type of forced symmetry-breaking is known to lead to rich dynamics, ${ }^{19-21}$ and the present system is no exception. To this end we note that the dominant conservative term that breaks the symmetry $(A, B) \rightarrow\left(e^{-i \phi} A, e^{i \phi} B\right)$ arising from rotation invariance of the system takes the form $i \Lambda(B, A)$, where $\Lambda$ is a real coefficient; the addition of this term to the equations breaks rotational invariance, but preserves a plane of reflection symmetry. In the following we assume that $\Lambda \sim \delta \sim d \sim \mu$, and project the streaming flow onto the first hydrodynamic mode. This assumption requires that the effective Reynolds number (42) of the streaming flow be sufficiently small that the nonlinear terms in (33) may be neglected. In the following we denote the (real) amplitude of this mode by $v_{1}$. We obtain $^{22}$

$$
v_{1}^{\prime}(\tau)=\varepsilon\left(-v_{1}+|B|^{2}-|A|^{2}\right),
$$




$$
\begin{aligned}
& A_{t}=\left[-\delta-i d+i\left(\alpha_{3}|A|^{2}-\alpha_{4}|B|^{2}\right)\right] A+i \alpha_{5} \mu \bar{B}+i \Lambda B-i \gamma v_{1} A, \\
& B_{t}=\left[-\delta-i d+i\left(\alpha_{3}|B|^{2}-\alpha_{4}|A|^{2}\right)\right] B+i \alpha_{5} \mu \bar{A}+i \Lambda A+i \gamma \nu_{1} B,
\end{aligned}
$$

where $\varepsilon=-\lambda_{1} R e^{-1}>0$ and $\lambda_{1}<0$ is the first hydrodynamic eigenvalue. These equations illustrate well the interplay between breaking of rotation invariance $(\Lambda \neq 0)$ and the excitation of streaming flow $(\gamma \neq 0)$. When $\Lambda=\gamma=0$ they reduce to the exact, but decoupled, amplitude equations for $A$ and $B$ that follow from Equations (41) on using (38).

Equations (45)-(47) have solutions in the form of reflection-symmetric standing waves $(A, B) \equiv(C, C), v_{1}=0$, where

$$
\delta^{2}+\left[d-\Lambda-\left(\alpha_{3}-\alpha_{4}\right)|C|^{2}\right]^{2}=\alpha_{5}^{2} \mu^{2}, \alpha_{3}-\alpha_{4} \neq 0
$$

this state sets in at $\mu=\mu_{c}$, where

$$
\mu_{c} \equiv \frac{\left[\delta^{2}+(d-\Lambda)^{2}\right]^{1 / 2}}{\alpha_{5}} .
$$

The amplitude $R \equiv|C|$ increases monotonically for $\mu>\mu_{c}$ provided $(d-\Lambda) /\left(\alpha_{3}-\alpha_{4}\right)$ $\leq 0$; if $(d-\Lambda) /\left(\alpha_{3}-\alpha_{4}\right)>0$ the branch bifurcates subcritically at $\mu=\mu_{c}$ before turning around towards larger $\mu$ at a secondary saddle-node bifurcation.

To determine the linear stability of these states we replace $A, B$, and $v_{1}$ by $C+$ $X_{ \pm} e^{\lambda t}+\bar{Y}_{ \pm} e^{\bar{\lambda} t}$ and $Z e^{\lambda t}+$ c.c., respectively, and linearize. The resulting equations have even eigenmodes $\left(X_{+}=X_{-}, Y_{+}=Y_{-}, Z=0\right)$ and $o d d$ eigenmodes $\left(X_{+}=-X_{-}\right.$, $Y_{+}=-Y_{-}, Z \neq 0$ ). The former preserve the reflection symmetry of $C$ and are characterized by the dispersion relation

$$
(\lambda+\delta)^{2}+\left[d-\Lambda-2\left(\alpha_{3}-\alpha_{4}\right)|C|^{2}\right]^{2}=\delta^{2}+(d-\Lambda)^{2} .
$$

Such instabilities are, therefore, always nonoscillatory and correspond either to the primary bifurcation at $\mu=\mu_{c}$ or to the secondary saddle-node bifurcation at

$$
|C|^{2}=\frac{d-\Lambda}{\alpha_{3}-\alpha_{4}} .
$$

In contrast, the symmetry-breaking perturbations satisfy a cubic dispersion relation,

$$
\lambda^{2}+2 \delta \lambda+4 d \Lambda-8 \Lambda\left[\frac{\gamma \varepsilon}{\lambda+\varepsilon}+\alpha_{3}\right]|C|^{2}=0,
$$

with a steady state bifurcation when

$$
|C|^{2}=\frac{d}{2\left(\alpha_{3}+\gamma\right)}, \text { if } d \Lambda \neq 0
$$

and a Hopf bifurcation when

$$
|C|^{2}=\frac{4 \Lambda d+2 \delta \varepsilon+\varepsilon^{2}}{4 \Lambda\left(2 \alpha_{3}-\delta^{-1} \varepsilon \gamma\right)}>0 .
$$

The oscillations that result are invariant under reflection followed by evolution in time by half the oscillation period $2 \pi /\left|\lambda_{l}\right|$, where

$$
\lambda_{I}^{2}=-\varepsilon^{2}-4 \delta^{-1} \varepsilon \gamma \Lambda|C|^{2}>0,
$$

and are the analogues of the direction-reversing waves discussed in the preceding section. Such symmetric oscillations can, therefore, be present only if 


$$
\gamma \Lambda<0,
$$

and so cannot occur near $\mu=\mu_{c}$ without both the streaming flow and the breaking of the rotation invariance of the system. This is a consequence of the low Reynolds number assumption used to model the streaming flow by a single mode. Recall that FIGure 4 shows that if Re is sufficiently large a symmetry-breaking Hopf bifurcation can destabilize the standing waves even if $\Lambda=0$.

From Equations (51), (53), and (54) we also find conditions for codimension-two degeneracies:

1. A Takens-Bogdanov bifurcation, corresponding to the coalescence of the symmetry-breaking Hopf and steady bifurcations, occurs if (56) holds and

$$
\left(\gamma+\alpha_{3}\right) \varepsilon+2 \delta^{-1} d \gamma \Lambda=0
$$

2. An interaction between the saddle-node and symmetry-breaking steady bifurcation occurs when

$$
d\left(\alpha_{3}+\alpha_{4}+2 \gamma\right)=2 \Lambda\left(\alpha_{3}+\gamma\right) ;
$$

3. An interaction between the saddle-node and symmetry-breaking Hopf bifurcation, with one zero plus two nonzero imaginary eigenvalues, occurs when (55) holds and

$$
\frac{4 \delta \Lambda+2 \delta \varepsilon+\varepsilon^{2}}{4 \Lambda\left(2 \alpha_{3}-\delta^{-1} \varepsilon \gamma\right)}=\frac{d-\Lambda}{\alpha_{3}-\alpha_{4}} .
$$

The first of these bifurcations contains within its unfolding periodic oscillations that are symmetric in the sense defined above. The second also contains periodic oscillations but this time the oscillations are asymmetric. Finally, the third bifurcation contains quasiperiodic solutions ${ }^{23}$ that are symmetric on average; these correspond to three-frequency states in the Faraday system. Chaotic dynamics are present near global bifurcations with which the quasiperiodic solutions terminate. ${ }^{24,25}$ Closely related results apply to containers subjected to horizontal vibration as well. ${ }^{26}$

\section{CONCLUDING REMARKS}

In this paper we have reexamined the theory of parametrically excited surface gravity-capillary waves in nearly inviscid liquids; the corresponding microgravity results are obtained on setting the Bond number $B$ to zero $\left(C_{g}=C, S=1\right)$. We focused on brimful containers with a pinned contact line and restricted attention to twodimensional systems with periodic boundary conditions. In systems of this type the primary instability is always to a pattern of standing waves - this is a very general result. ${ }^{27}$ In the present case, we have found that these standing waves may lose stability at finite amplitude to two different types of instabilities that break the reflection symmetry of these waves: a Hopf bifurcation producing standing waves that drift back and forth, and a parity-breaking bifurcation that produces waves that drift with a constant speed in one direction or other. We have shown that these instabilities involve the excitation of a viscous mean flow we called streaming flow. This flow is driven in the boundary layers at the container walls or at the free surface by time-averaged Reynolds stresses produced by the waves, and in turn couples to the 
amplitudes of the waves responsible for it. This coupling arises already at third order indicating that it is in general inconsistent to ignore the streaming flow while retaining other cubic terms. We have shown that for both extended $(L>1)$ and small $(L \sim 1)$ domains the general coupled amplitude-mean flow (GCAMF) equations decouple into equations for the wave amplitudes, and a set of equations for the spatial phase of the waves coupled to the Navier-Stokes equation for the streaming flow. In an extended domain the dynamics in the decoupled amplitude equations can be complex, and could break, at large enough forcing, the instantaneous equality $\left|A_{0}\right|=\left|B_{0}\right|$ characteristic of standing waves (FIG. 3); in a small domain this is not possible and standing waves persist for all time. However, in both cases the coupling to the streaming flow could destabilize the spatial phase of the standing waves resulting in complex drift motion. Our calculations suggest that the uniformly drifting standing waves observed in some experiments ${ }^{14}$ could form as a result of a parity-breaking bifurcation of the type described here. We have presented a concrete example of the two types of drift instability that can occur, and described briefly the interaction between the excitation of the streaming flow and forced breaking of the translation invariance of the (annular) container that suggests a variety of new experiments on the Faraday system. These conclusions readily generalize to containers of other shapes, such as square containers perturbed to rectangular ones. ${ }^{22}$

\section{ACKNOWLEDGMENTS}

We are grateful to María Higuera and Elena Martín for assistance in obtaining the results reported here, and to the NASA Microgravity Program for support under grant NAG3-2152.

\section{REFERENCES}

1. Miles, J. \& D. Henderson. 1990. Parametrically forced surface waves. Annu. Rev. Fluid Mech. 22: 143-165.

2. Fauve, S. 1995. Parametric instabilities. In Dynamics of Nonlinear and Disordered Systems. G. Martínez Mekler \& T.H. Seligman, Eds.: 67-115. World Scientific.

3. Kudrolli, A. \& J.P. Gollub. 1997. Patterns and spatio-temporal chaos in parametrically forced surface waves: a systematic survey at large aspect ratio. Physica D 97: $133-154$.

4. Martel, C. \& E. Knobloch. 1997. Damping of nearly inviscid water waves. Phys. Rev. E 56: 5544-5548.

5. VeGa, J.M., E. Knobloch \& C. Martel. 2001. Nearly inviscid Faraday waves in annular containers of moderately large aspect ratio. Physica D 154: 313-336.

6. LAMB, H. 1932. Hydrodynamics. Cambridge University Press.

7. Ezerskit, A.B., M.I. Rabinovich, V.P. Reutov \& I.M. Starobinets. 1986. Spatiotemporal chaos in the parametric excitation of a capillary ripple. Sov. Phys. JETP 64: $1228-1236$

8. Miles, J. 1993. On Faraday waves. J. Fluid Mech. 248: 671-683.

9. Pierce, R.D. \& E. KNoBloch. 1994. On the modulational stability of traveling and standing water waves. Phys. Fluids 6: 1177-1190.

10. HAnsen, P.L. \& P. Alstrom. 1997. Perturbation theory of parametrically driven capillary waves at low viscosity. J. Fluid Mech. 351: 301-344.

11. DAVEY, A. \& K. STEWARTSON. 1974. On three-dimensional packets of surface waves. Proc. R. Soc. London, Ser. A 338: 101-110. 
12. Longuet-Higgins, M.S. 1953. Mass transport in water waves. Phil. Trans. R. Soc. Ser. A 245: 535-581.

13. Schlichting, H. 1932. Berechnung ebener periodischer Grenzschichtströmungen. Phys. Z. 33: 327-335.

14. Douady, S., S. Fauve \& O. Thual. 1989. Oscillatory phase modulation of parametrically forced surface waves. Europhys. Lett. 10: 309-315.

15. Martel, C., E. Knobloch \& J.M. VEGa. 2000. Dynamics of counterpropagating waves in parametrically forced systems. Physica D 137: 94-123.

16. Higuera, M., J. Porter \& E. KNobloch. 2002. Heteroclinic dynamics in the nonlocal parametrically driven Schrödinger equation. Physica D. 162: 155-187.

17. Martín, E., C. Martel \& J.M. Vega. 2002. Drift instability of standing Faraday waves. J. Fluid Mech. In press.

18. Landsberg, A.S. \& E. Knobloch. 1991. Direction-reversing traveling waves. Phys. Lett. A 159: 17-20.

19. Dangelmayr, G. \& E. Knobloch. 1991. Hopf bifurcation with broken circular symmetry. Nonlinearity 4: 399-427.

20. Dangelmayr, G., E. Knobloch \& M. Wegelin. 1991. Dynamics of travelling waves in finite containers. Europhys. Lett. 16: 723-729.

21. Hirschberg, P. \& E. Knobloch. 1996. Complex dynamics in the Hopf bifurcation with broken translation symmetry. Physica D 90: 56-78.

22. Higuera, M., J.M. VeGa \& E. KnOBloch. 2002. Coupled amplitude-streaming flow equations for nearly inviscid Faraday waves in small aspect ratio containers. J. Nonlinear Sci. In press.

23. Guckenheimer, J. \& P. Holmes. 1983. Nonlinear Oscillations, Dynamical Systems and Bifurcations of Vector Fields. Springer-Verlag.

24. LANGFORD, W.F. 1983. A review of interactions of Hopf and steady-state bifurcations. In Nonlinear Dynamics and Turbulence. G.I. Barenblatt, G. Iooss \& D.D. Joseph, Eds.: 215-237. Pitman.

25. KiRK, V. 1993. Merging of resonance tongues. Physica D 66: 267-281.

26. Higuera, M., J.A. Nicolás \& J.M. VEGA. 2002. Weakly nonlinear oscillations of nonaxisymmetric capillary bridges at small viscosity. Phys. Fluids. In press.

27. Riecke, H., J.D. Crawford \& E. KNobloch. 1988. Time-modulated oscillatory convection. Phys. Rev. Lett. 61: 1942-1945. 